\title{
Multiwavelength variability analysis of the FSRQ 3C 279
}

\author{
V. Patiño-Álvarez ${ }^{1}$, V. Chavushyan ${ }^{1}$, J. León-Tavares ${ }^{1}$, \\ A. Carramiñana ${ }^{1}$, L. Carrasco ${ }^{1}$, S. Fernandes ${ }^{2}$, E. M. Schlegel $^{2}$ \\ and E. López-Rodríguez ${ }^{2}$ \\ ${ }^{1}$ Instituto Nacional de Astrofísica, Óptica y Electrónica (INAOE), Puebla, México \\ email: victorp@inaoep.mx \\ ${ }^{2}$ University of Texas at San Antonio, Department of Physics and Astronomy, \\ One UTSA Circle, San Antonio, TX 78249, USA
}

\begin{abstract}
We present a multifrequency analysis of the variability in the flat-spectrum radio quasar 3C 279 from 2008 to 2014. Our multiwavelength dataset includes gamma-ray data from Fermi/LAT (Abdo et al. 2009), observations in 1mm from SMA (Gurwell et al. 2007), Near Infrared from OAGH (Carramiñana \& Carrasco 2009) and SMARTS (Bonning et al. 2012); optical V band from the Steward Observatory (Smith et al. 2009) and SMARTS; optical spectra from OAGH (Patiño-Álvarez et al. 2013) and the Steward Observatory; and polarization spectra from the Steward Observatory. The light curves are shown in Fig. 1. Six out of seven optical activity periods identified within our dataset show clear counterparts in mm, NIR and gammarays, however, the late 2011 - early 2012 optical flare does not have a counterpart in the $\mathrm{GeV}$ regime. In this contribution, we discuss the flaring evolution of 3C 279 and speculate about the production of the anomalous activity period.
\end{abstract}

Keywords. galaxies: active, galaxies: jets, gamma rays: galaxies, quasars: individual (3C 279)

\section{Introduction}

3C 279, a source at $\mathrm{z}=0.536$ was one of the first gamma-ray quasars discovered with the Compton Gamma-Ray Observatory (Hartman et al. 1992). It has long been known that the emission of this source is highly variable, from the radio, optical, up to $\gamma$-rays. Therefore, several intensive multi-wavelength campaigns (see, e.g., Abdo et al. 2010, Bonning et al. 2012) and theoretical studies (e.g., Böttcher \& Principe 2009) have led to some important conclusions about the physical properties of 3C 279. Despite those studies, a general consensus about the location of the gamma-ray production zone in $3 \mathrm{C}$ 279 does not yet exist.

\section{Correlation analysis}

Using cross-correlation analysis we have found zero delay among the UV $\lambda 3000 \AA$ continuum emission, the optical V band, and the NIR J, H and K bands. This correlation allows us to speculate that the emission from the middle UV range to the NIR is emitted in the same region. Based on this, every correlation we find with the $\lambda 3000 \AA$ continuum emission is assumed to exist also with the $\mathrm{V}$ and NIR bands. We report the finding of a significant correlation $(\rho=0.65, \mathrm{P}<<0.01)$ between the $\lambda 3000 \AA$ continuum emission and $1 \mathrm{~mm}$ emission during our entire observation time range. This strongly suggests that the UV continuum emission is dominated by non-thermal emission from the jet. These results strongly suggest that the continuum emission from the UV to the NIR bands, during the observation period, share the same non-thermal origin. The highly variable 


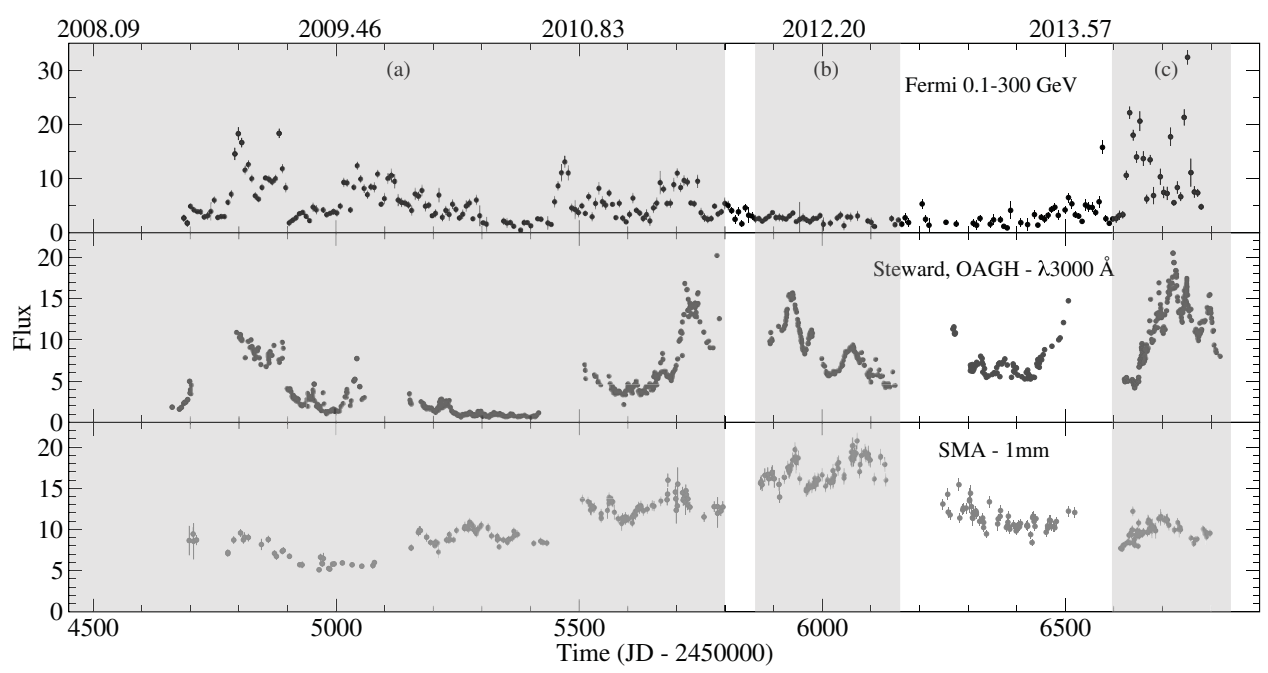

Figure 1. Multiwavelength light curves for 3C 279. Figure units are: Top panel $\left[\mathrm{ph} \mathrm{cm}^{-2} \mathrm{~s}^{-1}\right]$; middle panel $\left[\times 10^{-15} \mathrm{erg} \mathrm{cm}^{-2} \mathrm{~s}^{-1} \AA^{-1}\right]$; bottom panel [Jy].

levels of optical degree of polarization shown during the different flares further strengthen the idea of the optical continuum (and therefore UV and NIR) having a non-thermal origin. The cross-correlation analysis shows a delay of $-8.7 \pm 13.4$ days between the $\lambda 3000 \AA$ continuum emission and the gamma-rays during period "a". However, during period "c" (see Figure 1) we find a delay of $-72.6 \pm 8.8$ days among the same bands.

\section{Anomalous activity period}

We report the finding of an anomalous activity period in the light curves of $3 \mathrm{C} 279$. In this period we find two flares in the $\lambda 3000 \AA$ continuum with counterparts in the optical $\mathrm{V}$ and NIR bands, as well as the highest flux levels of $1 \mathrm{~mm}$ emission during our entire time range. However, there is no counterpart in the gamma-rays to any of these two flares (see period "b" in Figure 1). A similar behavior has been previously reported for the source PKS 0208-512 (Chatterjee et al. 2013) where they speculate this was caused by a change in the magnetic field without any change in the total number of emitting electrons or Doppler factor of the emitting region.

The reason behind the different delays that we find in our light curves (period "a" and "c"), and the lack of a counterpart in gamma-rays in period "b" is unclear; however we are still investigating the nature of this peculiar flaring behavior.

\section{References}

Abdo, A. A., Ackermann, M., Ajello, M., et al. 2009, ApJS, 183, 46

Abdo, A. A., Ackermann, M., Ajello, M., et al. 2010, Nature, 463, 919

Bonning, E., Urry, C. M., Bailyn, C., et al. 2012, ApJ, 756, 13

Böttcher, M. \& Principe, D. 2009, ApJ, 692, 1374

Carramiñana, A. \& Carrasco, L. 2009, RMxAC, 37, 132

Chatterjee, R., Fossati, G., Urry, C. M., et al. 2013, ApJL, 763, L11

Gurwell, M. A., Peck, A. B., Hostler, S. R., et al. 2007, ASP Conf. Ser., 375

Hartman, R. C., Bertsch, D. L., Fichtel, C. E., et al. 1992, ApJL, 385, L1

Patiño-Álvarez, V., et al. 2013, 4th Fermi Symposium, eConf Proceedings C121028

Smith, P. S, et al. 2009, Fermi Symposium, eConf Proceedings C091122 